\title{
Archéopages
}

Archéopages

Archéologie et société

37 | 04/2013

Jardins

\section{L'adaptation du modèle italique des « jardins » dans la Gaule romaine. Quelques exemples récemment fouillés en France}

Véronique Brunet-Gaston

\section{OpenEdition}

Journals

Édition électronique

URL : https://journals.openedition.org/archeopages/337

DOI : $10.4000 /$ archeopages.337

ISSN : 2269-9872

Éditeur

INRAP - Institut national de recherches archéologiques préventives

Édition imprimée

Date de publication : 1 février 2014

Pagination : 10-17

ISSN : $1622-8545$

Référence électronique

Véronique Brunet-Gaston, «L'adaptation du modèle italique des « jardins » dans la Gaule romaine. Quelques exemples récemment fouillés en France », Archéopages [En ligne], 37 | 04/2013, mis en ligne le 01 avril 2015, consulté le 21 janvier 2022. URL : http://journals.openedition.org/archeopages/337 ; DOI : https://doi.org/10.4000/archeopages.337 


\title{
L'adaptation du modèle italique des «jardins » dans la Gaule romaine Quelques exemples récemment fouillés en France
}

\author{
Véronique Brunet-Gaston Irrap, USR 3155 , IRAA
}

Devant le portique, une promenade est scandée par les plates-bandes de buis taillées de façons diverses. Plus bas descend doucement un lit de gazon, sur lequel le buis dessine des figures de bêtes féroces qui s'affrontent. En bas, il y a de souples figures d'acanthe, presque ondoyantes...

Pline le Jeune, Lettres, V, 6, 14-23 (Adam, 2012).

1. Sur les six éléments autunois qui devaient tous posséder des piètements épais, aucun ne subsiste, à l'exception d'un trapézophore dont les proportions et le matériau (marbre de Carrare) nous incitent à le considérer comme une importation italique.
Si la culture des fleurs est attestée en Grèce dès la fin du $\mathrm{v}^{\mathrm{e}}$ siècle avant notre ère, le jardin romain (hortus) n'apparaît qu'au II $^{\mathrm{e}}$ siècle avant notre ère ; alors voué aux besoins quotidiens, il correspond à notre potager, avec des arbres fruitiers. Le jardin planté d'arbres, le viridari, devient ainsi un espace de repos et donne naissance à un véritable art du jardin. En Gaule romaine, une tradition de mise en scène de l'eau - repérable dès le début $\mathrm{du} \mathrm{I}^{\mathrm{er}}$ siècle avant notre ère - est favorisée, à partir de l'époque augustéenne, par la création de réseaux de distribution hydraulique sous pression dans de nombreuses agglomérations. Le mobilier en calcaire ou en marbre mis au jour lors des opérations archéologiques - qui n'est pas toujours exploité à sa juste valeur - témoigne de la richesse de ces aménagements. Si les dernières publications sur l'habitat en Gaule Belgique et en Germanie montrent le rôle important des jardins et des décors de fontaines dans les zones septentrionales (Dessales, 2011), des sites archéologiques répartis dans presque tout l'eśpace français ont ainsi révélé des décors luxueux, évoquant les principes de l'amoenitas (Bedon, 2002 et 2007-2008).

La découverte récente, à Clermont-Ferrand, de nombreux vestiges de vasques et d'une fontaine à escalier d'eau offre l'occasion d'évoquer les techniques d'adduction, mais aussi les éléments de décor qui contribuaient à la scénographie de ces lieux de promenade et de délassement, et de les comparer aux demeures pompéiennes.
Les bassins et les vasques

Les bassins, attributs utilitaires et ornementaux du paysage urbain (Agusta-Boulardot, 2008a et 2008b), s'imposent dans l'habitat romain dès le milieu du $\mathrm{II}^{\mathrm{e}}$ siècle avant notre ère en association avec le péristyle (Vipard, 2007, et Dessales, 2011, p. 242). Les jardins des plus riches demeures pompéiennes conservent encore des vasques en place avec jet d'eau comme dans la domus des Vettii, où l'enfilade des bassins est quasiment en état de fonctionnement. En France, ces vasques bien étudiées en Italie (Ambrogi, 1995, p. 14-18, type A II) et les fonticuli (fontaines à escaliers d'eau où l'eau s'écoule depuis une petite réserve sur trois ou quatre escaliers présents sur les faces) s'avèrent fort rares. Seuls quelques spécimens de bassins ou de vasques sont connus. Il est probable que, dans nos provinces, leurs vestiges, trop fragmentaires, soient encore mal interprétés (apparentés à des corniches, par exemple) ou indéterminés : un travail d'identification systématique de ce type de mobilier dans les réserves archéologiques serait donc indispensable (Gaston, 2007). Des bassins et vasques ont cependant été retrouvés lors des fouilles d'Autun, Meaux, Clermont-Ferrand ou Bergheim. Lanalyse menée par Christophe Gaston sur les vasques éduennes, ${ }^{1}$, comparées à celles de Pompéi, montre comment, de l'importation d'artefacts italiques, on passe à la création de modèles en pierre locale imitant des marbres prestigieux (Gaston, 2007 ; Brunet-Gaston, 2010). 

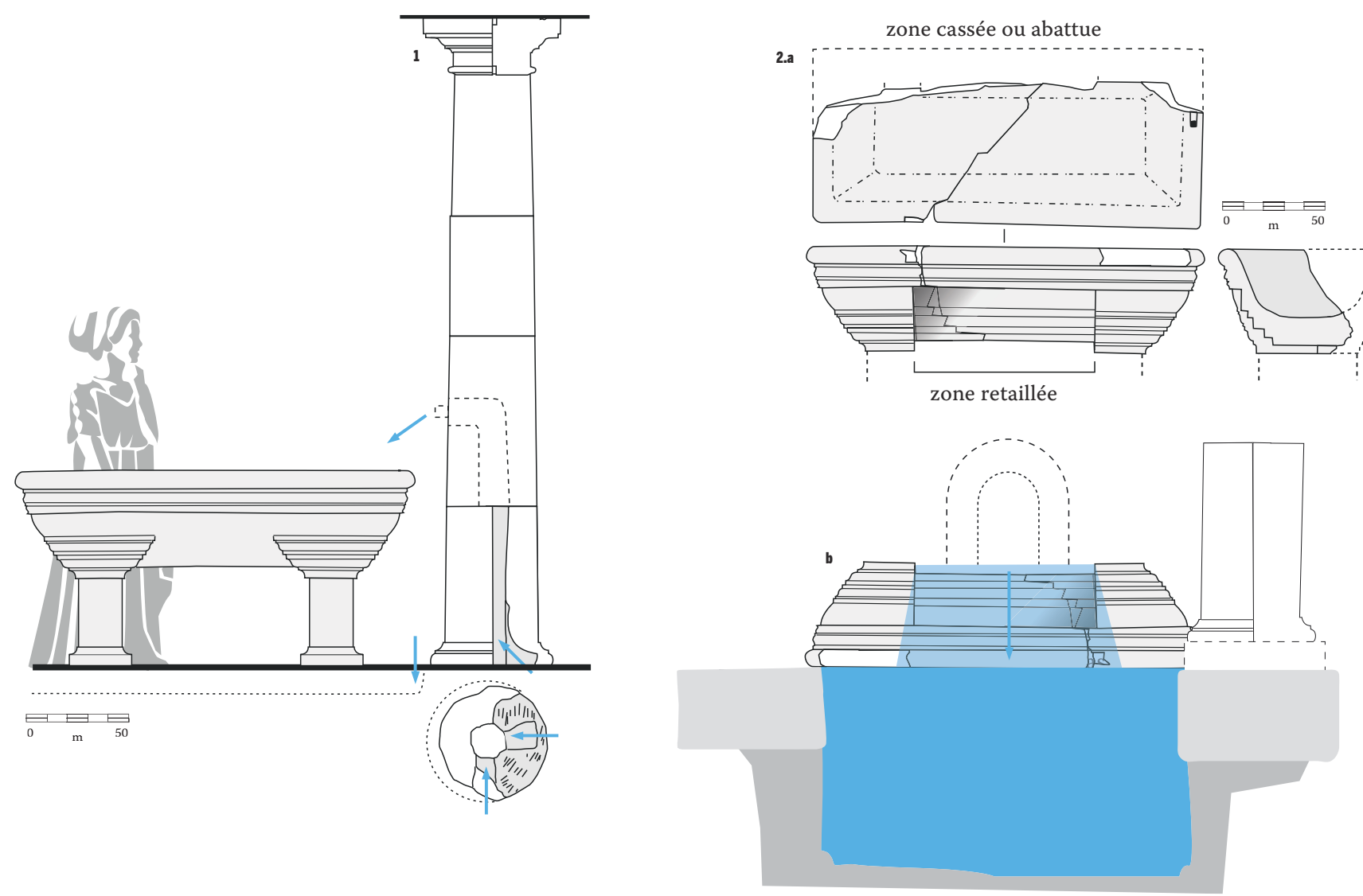

1. Cette reconstitution montre la vasque de Clermont-Ferrand (Carré Jaude II) dans son état initial et la proposition de circuit de l'eau

à l'intérieur des fûts de colonne évidés. Le tambour est entièrement perforé d'une cavité

de $15 \mathrm{~cm}$ de diamètre pour le passage d'une fistula et des cavités de $8 \mathrm{~cm}$ de large (s'évasant) sont creusées dans la moulure de la base pour les adductions d'eau qui devaient courir dans le caniveau périphérique. Un tambour devait comporter des jets d'eau

intégrés qui alimentaient une ou deux vasques, dont le débordement était récupéré dans le caniveau.
$2 a$ et $b$. La grande vasque à deux pieds en dalle retrouvée à Clermont-

Ferrand (Carré Jaude II) a été brisée puis remployée en fontaine à escalier dans les latrines.

Réalisée en calcaire local bleu-gris de Ferrièressur-Sichon, elle imite le marbre bleu Turquin italique et rappelle les modèles importés d'Italie.

3. Plan des latrines intégrant la vasque réutilisée comme fontaine en cascade.
$\Lambda$

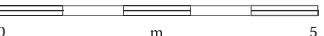

$\rightarrow$ Circulation de l'eau claire

Circulation des eaux usées

Vasque de marbre

Éléments de colonnes

Sols de béton
Conduite d'adduction d'eau

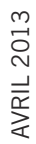

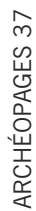



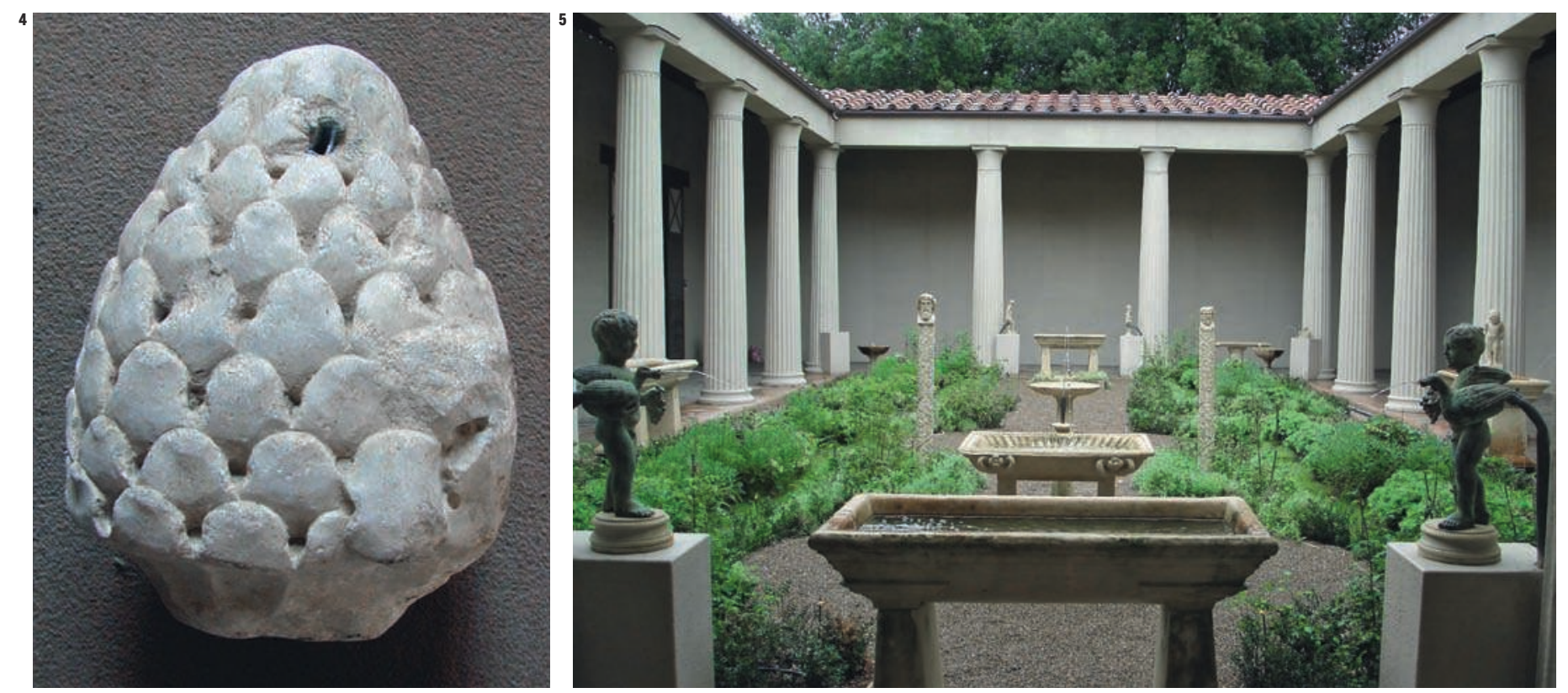

$6 . a$
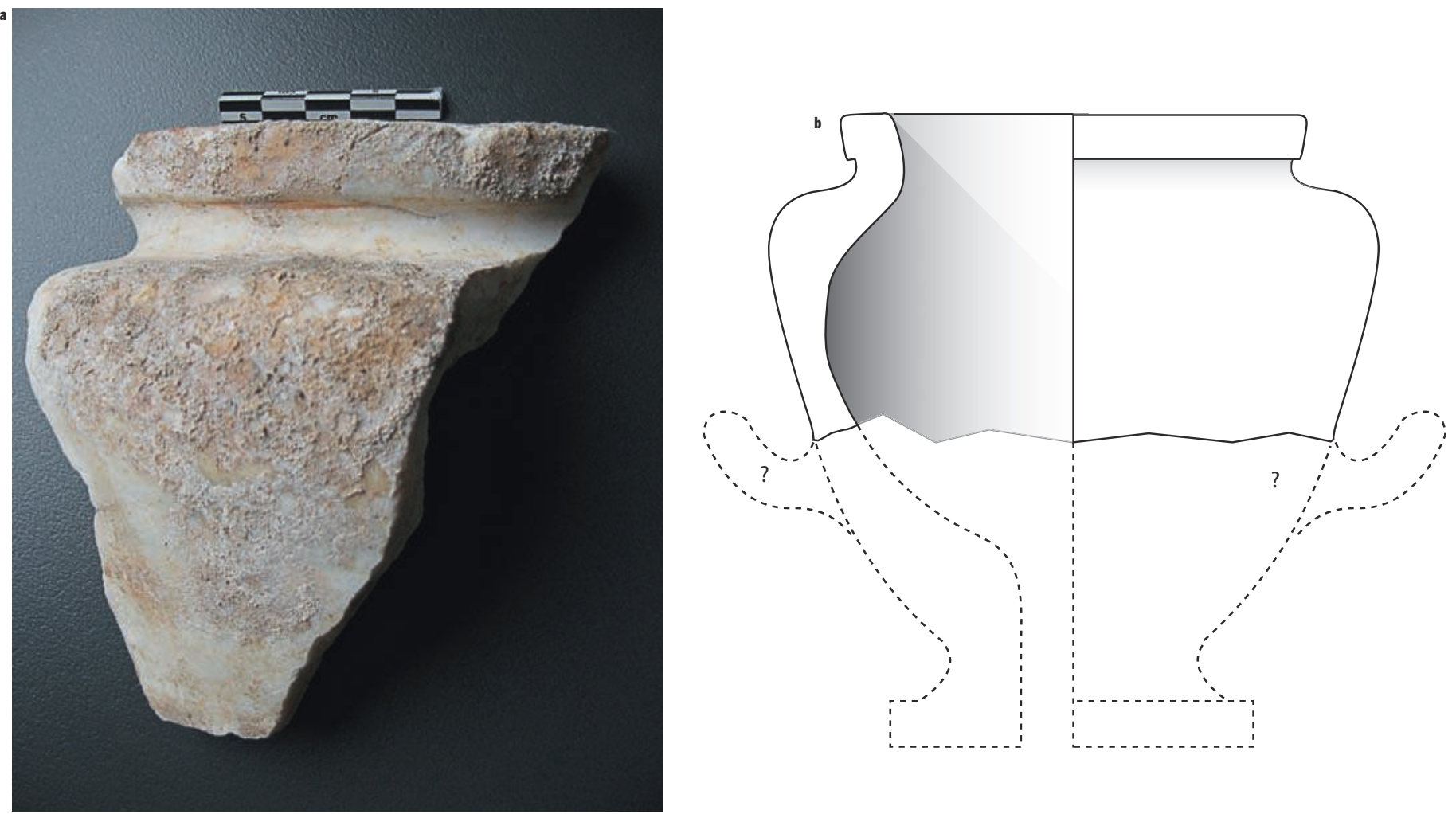

4. Le jet d'eau en marbre blanc de Carrare retrouvé à Autun figure un cône de pin formant l'extrémité d'un thyrse : percé de haut en bas d'un conduit pour l'alimentation en eau, il est fermé au sommet par un bouchage en plomb. Partant de ce percement axial, trois petites conduites en plomb formant jet d'eau débouchent sur trois côtés du sommet de la pomme de pin. La différence de traitement de la partie

arrière indique qu'il était adossé. Hauteur : $16 \mathrm{~cm}$.
5. Le jardin de la Maison des Vettii, à Pompéi est le parfait exemple de jardin d'agrément où le bruit des vasques et fontaines scandait les journées. On y trouve les statuettes-fontaines en pierre ou en bronze déversant un filet d'eau continu, ainsi que des colonnettes surmontées d'hermès double face, ornements récurrents des jardins antiques.
6. Un fragment de cratère ornemental en marbre (a) et la restitution du cratère (b) découvert à Besançon (fouille du Palais de justice)

II illustre le système décrit par Galliazzo (1979):

le canal vertical creusé dans le cratère montait

l'eau jusqu'au rebord,

l'eau s'écoulait ensuite dans un bassin qui une fois plein se déversait à son tour dans un bassin inférieur ou un caniveau. 
La série de vasques de l'immense domus peut-être même siège de corporation - du Carré Jaude II à Clermont-Ferrand ${ }^{2}$ (Alfonso et al., 2012 ) est révélatrice de ce phénomène d'appropriation de modèles italiques. Une petite trentaine de fragments de vasques rectangulaires de toutes tailles (caractéristiques avec leur mouluration qui couronne l'emplacement des piètements), retrouvés sur tout le site, placent la cité des Arvernes au premier rang des collections du monde romain. Malgré cela, un seul fragment de pied en dalle a été identifié. Un grand nombre de fragments de chancel appartenaient peut-être à des lacus (fontaine de rue), ce qui pourrait indiquer que les vasques ornaient, non seulement le jardin, mais aussi toute l'esplanade. Issue de la sphère privée ou semi-privée, une grande vasque, initialement installée sur deux pieds [ill. 1], a été remployée (suite à un bris volontaire ou involontaire) en fontaine à escalier [ill. 2a et b], l'eau remplissant un canal qui délimitait l'espace des latrines [ill. 3].

Les modèles italiques offrent des indices de datation pour ces deux états successifs. L'installation initiale de la vasque sur deux pieds en dalle renvoie à un modèle élaboré en Grèce (Deonna, 1938, p. 78-79), qui se diffuse dans la première moitié $\mathrm{du} \mathrm{I}^{\mathrm{er}}$ siècle de notre ère dans le monde romain. Leur développement, qui coïncide, à Rome, avec celui de l'importation des marbres colorés, s'inscrit dans la première moitié $\mathrm{du} \mathrm{II}^{\mathrm{e}}$ siècle et perdure jusqu'au début du siècle suivant (Gaston, 2007, Ambrogi, 1995, n. 1, p. 59-60).

Le type de bruit produit par la fontaine fournirait un autre indice, concernant cette fois la chronologie du remploi de la vasque. Les fontaines à escaliers, ou fonticuli, sont plutôt d'habitude de petits objets décoratifs (Lavagne, 1998) et leur chronologie reste floue. En effet, avant 79 à Pompéi, les fonticuli n'existaient pas car on leur préférait les fontaines à escalier unique, de type monumental, adossées à une paroi (sous Néron et les Flaviens, Galliazzo, 1979). Ce sont des sortes de grottesfontaines dont l'exemple le plus significatif serait celle de la maison de D. Octavius Quartio ou celle de Marcus Lucretius (IX. 3.5) ou encore celle de Méléagre (VI.9.12). L'eau s'y écoule avec fracas (fragor) en opposition au murmure de l'eau des fonticuli de Trajan. La mode n'est pourtant pas la seule raison de ce contraste : il faut peut-être aussi prendre en compte la taille relativement modeste de certaines domus de Pompéi. À Clermont, le système d'adduction devait avoir un grand débit et l'eau, sur les marches bien formées de cette vasque renversée, devait produire un bruissement relativement fort.

La vasque de Clermont serait donc installée,

2. Fouille réalisée en 2009-2010; responsable d'opération: Guy Alfonso, Inrap. 3. Fouille du Collège Lumière à Besançon (Doubs), réalisée en 2003-2004 ; responsable d'opération : Claudine Munier. dans son usage initial, autour de l'époque hadrianique. À cette période, elle est pourtant déjà remployée en fontaine à escalier. Aurait-elle été cassée avant ou lors de sa mise en œuvre? Elle est en tout cas réutilisée d'une façon fort ingénieuse dans les latrines. La retaille du corps de la vasque ne laisse parfois que cinq centimètres d'épaisseur sur les dix de la paroi... La référence au modèle des fontaines monumentales apparaît alors comme un archaïsme, qui trahit peut-être la culture italique du commanditaire.

\section{Les techniques d'adduction}

De la simple bouche d'eau agrémentée d'un protomé au portique, en passant par le bassin de puisage, les aménagements hydrauliques vont de pair avec la nature du commanditaire et de leur emplacement (intégration au sein de la sphère privée ou complexe édilitaire, aménagement urbanistique - Agusta-Boulardot, 2001, p. 221-224).

La hauteur moyenne - du sol au rebord - des vasques est d'environ $80 \mathrm{~cm}$ (soit presque la hauteur d'une table actuelle). Le système d'alimentation est toujours situé à l'extérieur avec de multiples jets d'eau obliques, plus rarement un seul, comme dans le péristyle de la maison des Vettii à Pompéi [ill. 5] (Gaston, 2007). Par ailleurs, comme on peut l'observer dans les demeures de luxe à Pompéi, les jets d'eau peuvent être directement aménagés dans les colonnes du péristyle, ainsi dans les maisons de M. Holconius Rufus (VIII.4.4) et de C. Cornelius Rufus (VIII.4.15). Les fûts de colonne de la fouille de Jaude II à Clermont-Ferrand comportent ce dispositif [ill. 2] ; le diamètre creusé à l'intérieur des tambours de grès arkosique devait laisser la place à des tuyaux de plomb ou fistulae (Brunet-Gaston, 2012). À Autun (Aqua, 2010), a été retrouvé un jet d'eau en marbre, en forme de cône de pin, percé de conduits en plomb pour l'alimentation en eau [ill. 4] ; il provient sans doute d'une fontaine ornant le jardin d'une riche domus à la symbolique bachique. Trouve-t-il son modèle dans l'énorme cône en bronze, d'environ 2,50 m de haut, des thermes d'Agrippa (fin du i ${ }^{\text {er }}$ siècle de notre ère), conservé dans la cour de la Pigna, au Musée du Vatican?

À Besançon', deux fragments de cratères ornementaux [ill. 6], en marbre blanc, montrent la richesse du décor des jardins de l'élite (Munier et al., 2007). À Autun, un cratère lisse et un à godrons présentent un percement cylindrique axial au niveau du pied qui nous permet de proposer la fonction de jet d'eau central de bassin, à l'instar du cratère central en marbre blanc de la villa d'Echternach d'où jaillissait l'eau recueillie par un bassin octogonal maçonné (Metzler, Zimmer, 1982, p. 39-50). Ces réservoirs en forme de cratère ou d'urne, qui couronnent notamment les fontaines à escalier, seraient une innovation antonino-séverienne (Galliazzo, 1979, p. 72).

Lévacuation de la vasque se fait par débordement, soit dans un bassin maçonné peu profond (environ $10 \mathrm{~cm}$ ), soit dans le caniveau périphérique du péristyle, soit à même le sol du jardin. Le fonctionnement par « débordement » est confirmé par les profondes saignées verticales d'érosion dues au ruissellement de trop-plein, sur les dalles de piètement de ces vasques, comme cela a pu être observé à Autun ( $c f$. supra). 
7.L'hermès double mis au jour à Autun (faubourg d'Arroux) provient d'un buste posé sur un support a. Le personnage barbu et âgé, doté de cornes de béliers et de petites oreilles d'ovin est identifiable à Jupiter Ammon, divinité libyenne initialement consultée par Alexandre le Grand (oracle). b. L'autre personnage est un jeune homme imberbe,

à la chevelure bouclée,

conservant une petite aile, que l'on peut identifie à Hermès-Mercure. Sur les boucles de cheveux, une trace de rouille pourrait indiquer qu'une chaîne (?) reliait cette colonnette à une autre. II pourrait s'agir d'un élément d'une barrière composée de supports verticaux ou hermuli. La mortaise devait servir à la fixation d'un élément décoratif indéterminé

(couronne, guirlandes) ou à la fixation de cet élément sur un piédouche (marbre blanc à grain très fin et brillant). Hauteur : $13,5 \mathrm{~cm}$, courant du $\mathrm{I}^{\mathrm{er}}$ siècle. Un protomé en calcaire, identique à ce Jupiter Ammon, a été découvert à Brescia et daté stylistiquement de la seconde moitié du ler siècle de notre ère (Casari, 2004, p. 38-39, pl.10, b; Frova, 1994, p. 345-364, fig. 6,1). Ce type d'objet est souvent retrouvé dans les petites épaves de la côte méditerranéenne. 8. Apollon découvert lors de la fouille "faubourg d'Arroux", à Autun. La position très déhanchée, est typique des œuvres grecques. Nous avons ici un Apollon citharède demi-nu, inspiré d'une copie romaine du II siècle, d'après un original de Praxitèle, I'Apollon « lycien » mentionné par Lucien de Samosate (Anacharsis, 7); on trouve des exemplaires similaires dans les jardins pompéiens. Calcaire blanc fin de la côte Chalonnaise. Hauteur : $25 \mathrm{~cm}$.

9. Une statuette de victoire au drapé flottant au vent a été retrouvée à Besançon (Collège Lumière).

La surface très érodée est difficilement identifiable, le bras gauche replié sur le ventre retient un drapé à la pliure du coude. Calcaire fin, type Vergennes. Époque hadrianique.
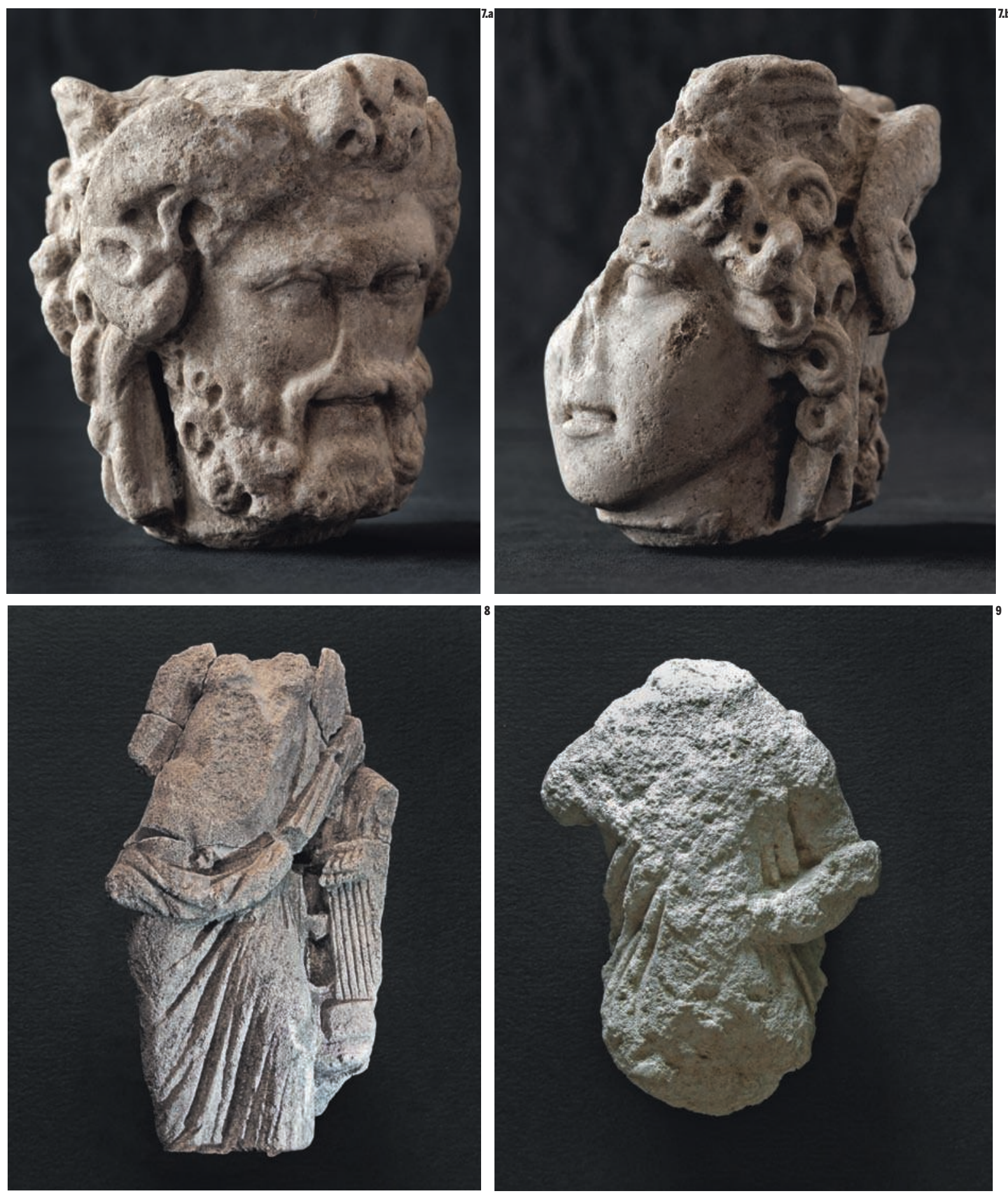
La parure en pierre : la statuaire et le mobilier

Outre les statues-fontaines zoomorphes (par exemple, Hermary, 2009), de rares témoignages nous montrent des décors raffinés même dans des contextes où on ne les attend pas. Les jardins sont agrémentés de statuettes, tel le double hermès [ill. 7], retrouvé lors de la fouille du site du faubourg d'Arroux à Autun', qui représente Mercure et Jupiter-Ammon. Son utilisation en tant que stèle hermaïque nous semble douteuse, surtout à cause de la retaille visible sur le cou. Il s'agit visiblement d'un artefact importé de Carrare (Italie). Le même site a livré une statuette d'Apollon à la lyre [ill. 8]. Le plan de l'îlot d'habitation et les vestiges mis en évidence relèvent de structures artisanales mêlées à de l'habitat modérément fortuné. Cependant le mobilier en pierre ne déparerait pas dans une riche demeure éduenne. Des éléments architecturaux tels que placages de marbre et éléments sculptés (corniches, vasques) sont présents, et de très beaux éléments statuaires importés devaient orner un jardin. Si le site autunois atteste que, même dans un quartier très modeste, on peut trouver une parure de qualité, à Besançon, la fouille du Collège Lumière (Munier et al., 2007) a permis la découverte d'une victoire ailée qui symbolisait peut-être la réussite du propriétaire de la somptueuse domus [ill. 9].

Des tables et banquettes participaient également à l'ornement des jardins. À Port-sur-Saône, des piètements zoomorphes figurent au sein du lapidaire de la villa du Magny ${ }^{\mathbf{5}}$ (Ribolet 2012, Brunet-Gaston et al., 2012). Il s'agit principalement de pattes de fauve sculptées et placées contre un élément vertical, fragments de sièges ou de petites banquettes aux montants décorés de protomés animaux. Des piètements similaires en roche locale ont été retrouvés à Autun (Brunet-Gaston et al., 2006, ML 898 et 899). Un pied mouluré de ce type, en calcaire local, a été découvert à Saint-Usage. $\mathbf{6}$ À Besançon, dans

\section{La mise en scène du jardin}

L'un des plus magnifiques exemples de jardins pompéiens est celui de la maison d'Octavius Quartio : traversé par un euripe (canal) orné d'édicules à fontaines, le jardin est couvert d'une pergola, et des treillages bordent les allées ; de longues haies d'arbustes sont régulièrement plantées. En France, plusieurs sites permettent d'évoquer diverses mises en scène associant la végétation à l'architecture, au mobilier, à la statuaire et aux jeux d'eau.

À Meaux, dans la domus dite au «nymphée privé », une fontaine occupe tout l'espace sous le portique de fond du jardin, en position centrale [ill. 10a]. L'eau tombée de la vasque à débordement, passant par une dalle en pierre perforée, se déversait dans un bac de décantation maçonné et dallé de tuiles, revêtu de mortier hydraulique [ill. 10b], puis rejoignait un bassin triangulaire qui la réinjectait dans un canal d'adduction. L'eau ressortait en jets par des aménagements inconnus, insérés dans le mur du fond, et retrouvait la vasque à débordement.

S'il est rare d'avoir un plan complet de l'organisation des espaces, le jardin était souvent visible depuis l'entrée de la maison. Les jeux d'eau se devaient aussi d'être observables depuis les salles de réception, lesquelles s'ouvraient généralement sur le péristyle. Outre leur vue plaisante, les jardins prodiguaient de la sorte fraîcheur et agrément sonore aux convives (Dessales, 2007, p. 107 et 251-252). La fontaine était ainsi à la fois le premier spectacle offert au visiteur par le propriétaire des lieux et un reflet de l'image sociale que ce dernier souhaitait renvoyer de lui-même (Dessales, 2007, p. 107). L'eau appartient en effet à un langage commun dans la sphère privée du monde romain, à tel point que même dans le décor peint, on retrouve des représentations de bassins ou de vasques peintes dans un écrin végétal (Barbet, 2008, p. 295-304) 9

La décoration des jardins passe aussi par des plantations soignées, en pot ou en pleine terre. La villa de Richebourg dans les Yvelines est un bel exemple de cette organisation paysagère.

Des plantations en pots perforés et enterrés se répartissaient sur une trame orthogonale d'environ 17 pieds (soit $5 \mathrm{~m}$ ), bordée probablement d'une rangée d'arbustes en abside. Cet ordonnancement se retrouve dans les jardins de la Villa Romana de Fréjus (fin du ${ }^{\text {er }}$ siècle avant notre ère-première moitié du $\mathrm{I}^{\mathrm{er}}$ siècle), sur un maillage orthogonal de 10 x 30 pieds (soit environ 3 x 9 m - Excoffon, 2011).

Ces espaces verts, souvent proches des salles ou des espaces de banquet, sont les composantes obligées d'un certain niveau de « standing ». Pourtant, compte tenu de nos climats continentaux, les pratiques du déjeuner au jardin devaient se limiter à quelques jours dans l'année ; le jardin gallo-romain est définitivement un lieu de paraître, même pour les habitats relativement modestes.

\footnotetext{
la fouille du Collège Lumière (Munier et al., 2007), deux plateaux de tables ont été mis au jour, l'un en calcaire et l'autre en calcite de grotte. D'un diamètre de $90 \mathrm{~cm}$ à $1 \mathrm{~m}$, ces tables devaient servir de desserte dans les salles de réception ou dans les jardins. À Reims, rue Maucroix ${ }^{7}$, le plateau de 6,5 cm d'épaisseur d'une table semi-circulaire en calcaire (diamètre estimé : $87 \mathrm{~cm}$ ) pouvait orner la grande cour de la domus de la parcelle sud (Rollet, 2009). À Meaux, boulevard Jean Rose, deux fragments de plateau de table moulurée de 5 à $6 \mathrm{~cm}$ d'épaisseur ont été trouvés. Des fragments de colonnettes toscanes d'un diamètre moyen de $20 \mathrm{~cm}$ sont associés à ces plateaux.

Si certaines de ces découvertes témoignent de l'importation d'artefacts produits dans les ateliers de sculpture proches de Carrare/Luni - commerce bien connu grâce notamment à l'étude du chargement des épaves -, d'autres illustrent les facultés d'adaptation et la technicité

des ateliers de sculptures locaux.

à septembre 2010 ;

Maucroix

(Marne), realiseer

responsable d'opération

8. Fouille du « boulevar

(Seine-et-Marne) réalisée

en 2007 ; responsable

d'opération : David

Couturier, Inrap.

9. Voir l'article d'Hélèn

Eristov et Florence

Monier p.18 de ce numéro.
} 


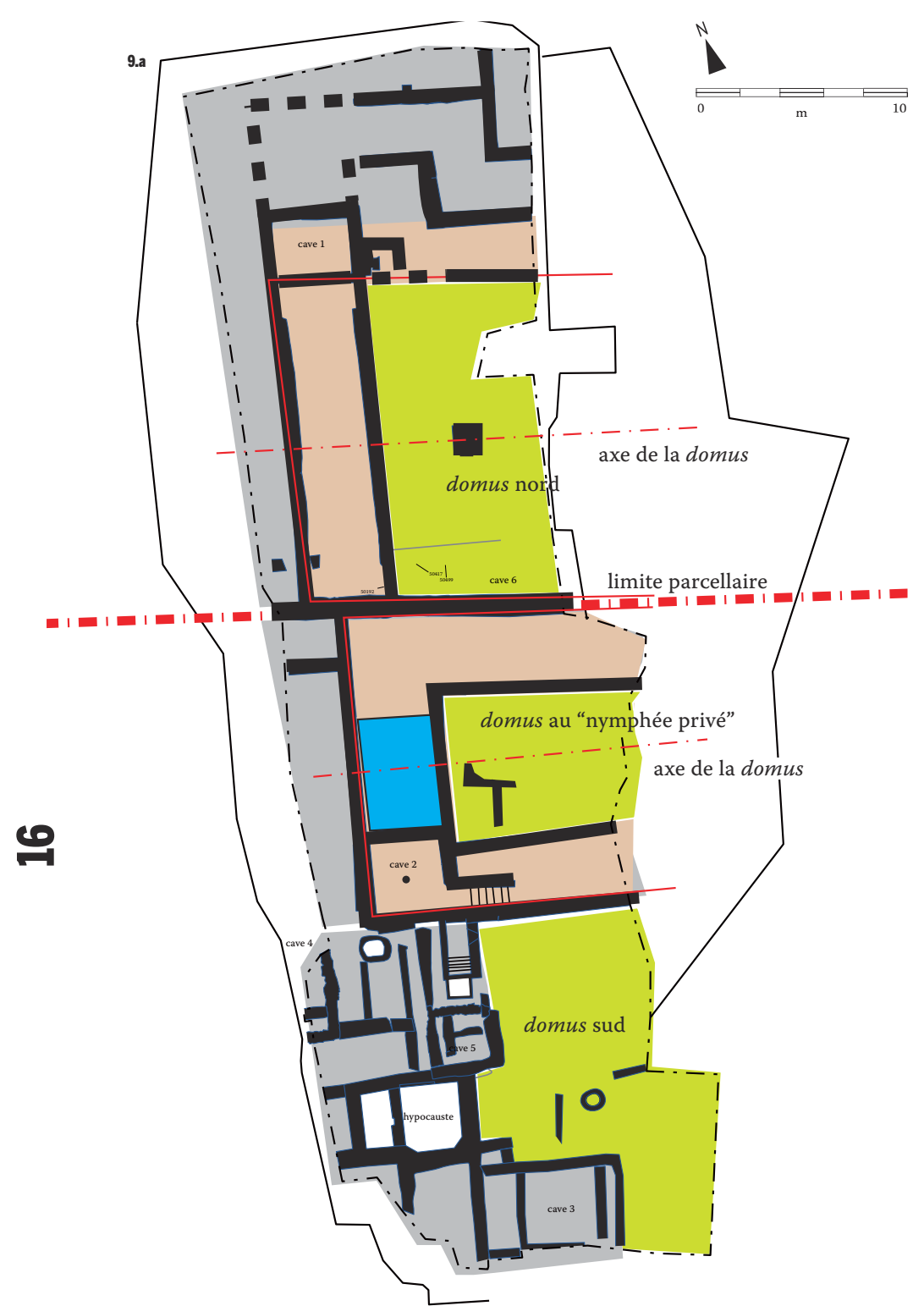

$10 \mathrm{a}$, b et c. Dans la domus longueur estimée à $58 \mathrm{~cm}$, au « nymphée privé ॥ (Meaux, boulevard pour une épaisseur

Jean Rose), des dalles de 6,5 à $7 \mathrm{~cm}$, comporte en calcaire de Paris et de Charenton de $25 \mathrm{~mm}$ d'épaisseur sont liées un léger surcreusement à l'intérieur d'une ciselure à l'aménagement du bass situé sous le portique, une dalle perforée sert de bonde pour l'évacuation des eaux. Un plateau de $42,5 \mathrm{~cm}$ de large et d'une

de $25 \mathrm{~mm}$ de large.

Sa surface est érodée par l'eau qui devait y ruisseler en permanence, peut-être s'agit-il d'un miroir d'eau qui recevait un jet d'eau.
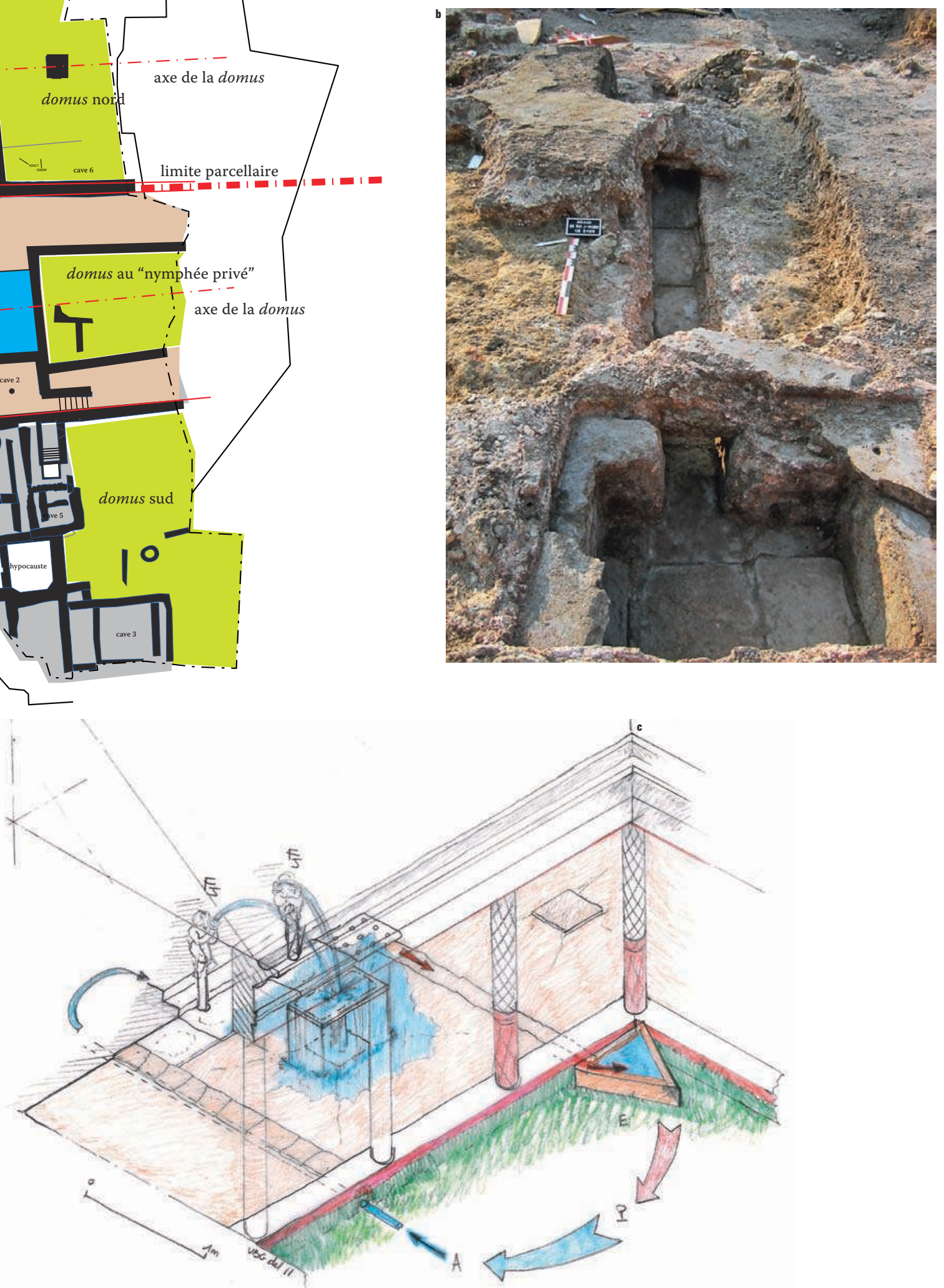


\section{Références bibliographiques}

ADAM J.-P., 2012, La maison romaine, Arles, H. Clair, 224 P

AGUSTA-BOULARDOT S., 2001, «Fontaines et fontaines monumentales en Grèce de la conquête romaine à l'époque flavienne : permanence ou renouveau architectural ? », in MARC J.-Y. et MORETTI J.-C. (dir), Constructions publiques et programmes édilitaires en Grèce entre le II ${ }^{e}$ s. av. J.-C. et le $\mathrm{I}^{e r}$ s. apr. J.-C., actes du colloque organisé par l'École française d'Athènes et le CNRS (Athènes, mai 1995), Athènes, BCH suppl. 39, p. 168-236.

Agusta-Boulardot S., 2008a, « Le lacus de la rue romaine : un exemple de "mobilier urbain" antique?», in Ballet P., Dieudonné-Glad N., Saliou C., La rue dans l'Antiquité: définition, aménagement et devenir, de l'Orient méditerranéen à la Gaule, Actes du colloque de Poitiers (septembre 2006), Rennes, Presses universitaires de Rennes, p. 93-100.

Agusta-Boulardot S., 2008b, «Dare aquas. Les enjeux politiques du "don de l'eau" dans les villes d'Italie républicaine ", Histoire Urbaine, 2008/2, p. 11-26.

Alfonso G. et al., 2012, Clermont-Ferrand (Puy-deDôme): ZAC de Jaude Ouest 2, rapport d'opération, Inrap-SRA Auvergne.

AMBROGI A., 1995, Vasche di età romana in marmi bianch e colorati, Rome, L'Erma di Bretschneider, 263 p.

AQUA, 2010, Aqua : la gestion de l'eau à Augustodunum, 30 juin-11 octobre 2010, ville d'Autun, Musée Rolin, petit journal de l'exposition, Autun, Musée Rolin, 11 p.

BARBET A., 2008, La peinture murale en Gaule romaine, Paris, Picard, $391 \mathrm{p}$

BEDON R. (éd.), 2002, Amoenitas urbium. Les agréments de la vie urbaine en Gaule romaine et dans les régions voisines, Limoges, Presses universitaires-PULIM, $491 \mathrm{p}$

BEDON R. (éd.), 2007-2008, Vicinitas Aquae, la vie au bord de l'eau en Gaule romaine et dans les régions voisines, Actes du colloque Caesarodunum XLI-XLII, Limoges, Presses universitaires-PULIM, $375 \mathrm{p}$.

BRUNET-GASTON V., 2010, «L'utilisation du marbre de Carrare dans les programmes monumentaux d'Autun au Haut-Empire », in CAMPOREALE $\mathrm{S}$ Dessales H. et Pizzo A. (éd.), Arqueología de la Construcción II. Los procesos constructivos en el mundo romano: Italia y provincias orientales, Mérida, Instituto de Arqueología de Mérida, p. 491-508.

BRUNET-GASTON V., 2012, «Étude du lapidaire architectural », in ALFONSO G. et al., 2012

Brunet-Gaston V., Blanc A. et P., ChardronPicault P., Gaston C., LAMotTe D., Lorenz I, TARDY D., 2006, rapport pluriannuel de Programme Commun de Recherche, « Pierres, techniques et décors architecturaux à Autun-Augustodunum », SRA Bourgogne, 2001-2006.

BRUNET-GASTON V.et al., 2012, Étude diachronique des vestiges subaquatiques en Haute-Saône, le cours de la Saône et ses aménagements : Port-sur-Saône (7o), DRAC de Franche-Comté.

CASARI P., 2004, Iuppiter Ammon e Medusa nell'Adriatico nordorientale : simbologia imperiale nella decorazione architettonica forense, Rome, Quasar (Studi e ricerche sulla Gallia Cisalpina 18 Antichità altoadriatiche, Monografie 1), $190 \mathrm{p}$.

DeONNA W., 1938, Le mobilier délien, Exploration archéologique de Délos, 18, Paris, E. de Boccard IV-4.07 p.

Dessales H., 2007, « Jeux d'eau et fontaines dans l'habitat de Pompéi », in Petit J.-P. et Santoro S. (dir), Vivre en Europe romaine, de Pompei à Bliesbruck Rheinheim, Paris, Errance, p. 103-108

Dessales H., 2011, « Le décor des fontaines domestiques en Gaule : un exemple d'adaptation des modèles italiques? », in BALMELle C., ERISTOV H. MONIER F. (éd.), Décor et architecture en Gaule entre l'Antiquité et le haut Moyen Âge : mosä̈que peinture, stuc (Actes du colloque international, 9-12 octobre 2008, Université de Toulouse II Le Mirail), Aquitania suppl. 2o, Bordeaux, p. 241-255
EXCOFFON P. (dir.), 2011, Ville et campagne de Fréjus romaine : la fouille préventive de "Villa Romana" Paris, Errance, Aix-en-Provence, Centre Camille Jullian (Bibliothèque d'archéologie méditerranéenne et africaine 8), $305 \mathrm{p}$

Frova A., 1994, « Tre sculture nel teatro romano di Brescia ", in SCARFi B. M., Studi di archeologia della $X$ regio in ricordo di Michele Tombolani, Rome, L'Erma di Bretschneider, p. 345-364.

Galliazzo V., 1979 « Significato e funzione della fontanella a scalette d'aqua nella casa romana ed un singolare frammento al Museo civico di Feltre », Atti della Accademia Roveretana degli agiati. Contributi della classe dio scienze umane, di lettere ed arti, 229, ser. VI, vol. 19, p. 49-82

GASTON C., 2007, « Vasques rectangulaires à pieds en dalle dans les collections d'Autun (Saône-et-Loire) : un mobilier en pierre méconnu ", Revue archéologique, 2007/2 ( $\left.{ }^{\circ} 44\right)$, p. 305-318.

Hermary A., 2009, « Un dauphin-fontaine trouvé dans le Puy-de-Dôme », in Gaggadis-Robin V. et al., Les ateliers de sculpture régionaux : techniques, styles et iconographie, Arles, Musée départemental Arles antique, p. 551-556.

JARDINS D'EMPÚRIES, 2005, Jardins d'Empúries. La jardineria en época romana, L'Escala, Museu d'arqueologia de Catalunya-Empúries, 120 p. (pour une orientation bibliographique sur les domaines connexes)

JASHEMSKI W. F., 1979 et 1993, The gardens of Pompeii : Herculaneum and the villas destroyed by Vesuvius, vol. I et II, New Rochelle, A. D. Caratzas, 372 et 432 p.

LAVAGNE H., 1998, « Fonticuli, deux fontaines à escaliers d'eau en Narbonnaise : Beaurepaire (Isère) et Nissanlez-Enserune (Hérault) », Revue des études anciennes, t. $100, n^{\circ} 1-2$, p. $269-287$

MeTZLER J., ZiMMER J., 1982, Echternach, une grande villa romaine du Luxembourg, Archeologia n 168, juillet 1982, p. 39-50.

MUNIER C. et al., 2007, Au collège Lumière, rapport d'opération, Inrap-SRA Franche-Comté.

Ribolet M., 2012, «Étude du mobilier lapidaire issu des fouilles anciennes de la villa du Magny », in BRUNET-GASTON V. et al., 2012.

Rollet P., 2009, Reims (Marne) - «17-19, rue Mont d'Arène et 6-8, rue Maucroix ", rapport d'opération, Inrap-SRA Champagne-Ardenne.

VIPARD P., 2007, « Maison à péristyle et élites urbaines en Gaule sous l'Empire », Gallia, 64, p. 227-277. 